Pacific Journal of Mathematics

CLOSED ORBITS OF CONVEX SETS OF OPERATORS ON THE 


\title{
CLOSED ORBITS OF CONVEX SETS OF OPERATORS ON THE DISK ALGEBRA
}

\author{
J. N. McDoNALD
}

Let $\mathscr{P}$ denote the set of linear transformations of the disk algebra which have norm one and fix the constants. In this paper the orbits of functions in the disk algebra under the action of $\mathscr{P}$ are described.

Let $\mathscr{A}$ denote the disk algebra, i.e., the algebra of functions which are analytic on the open unit disk $D$ and continuous on $\bar{D}$. Let $\mathscr{A}$ be equipped with the sup-norm $\|\cdots\|$ and let $\mathscr{C}$ denote the closed unit ball in $\mathscr{A}$. Consider the set $\mathscr{P}$ of linear operators which map $\mathscr{A}$ into itself, have norm 1 and fix the constants. $\mathscr{P}$ acts as a semi-group of transformations of the set $\mathscr{C}$. In this paper we study the closed orbits of functions in $\mathscr{C}$ under the action of $\mathscr{P}$, i.e., the sets

$$
\mathscr{P} f=\text { closure }\{T f \mid T \in \mathscr{P}\}
$$

for $f \in \mathscr{U}$. We will show that $\mathscr{P} f$ is the closed convex hull of the functions $[F, G] f$, where $F$ and $G$ range over the inner functions in $\mathscr{A}$. Here

$$
[F, G] g(z)=(2 \pi n i)^{-1} \int_{\partial D} g(\xi) F^{\prime}(\xi)(G(z)-F(\xi))^{-1} d \xi,
$$

where $n$ is the number of zeros of $F$ and $z \in D$. (Recall that the inner functions in $\mathscr{A}$ are exactly the finite Blaschke products, that is functions of the form

$$
F(z)=e^{i s} \prod_{j-1}^{n} \frac{z-\alpha_{j}}{1-\bar{\alpha}_{j} z}
$$

where $\left|\alpha_{j}\right|<1$ for $j=1,2, \cdots, n$.) We will also show that our result can be viewed as a generalization of a theorem due to Fisher [3]. The final section of the paper contains a discussion of the possibility of extending our results to the algebra $H^{\infty}$ of bounded analytic functions on $D$.

1. Background. Perhaps a few sentences should be devoted to the context of this work in the literature. In [9] Phelps asked for a description of the extreme points of the convex set $\mathscr{P}$. In the same paper he was able to characterize those extreme elements of $\mathscr{P}$ which happen to be multiplicative. They are exactly the 
operators of the form

$$
C_{\phi} f=f \circ \dot{\phi},
$$

where $\phi$ is an extreme element of $\mathscr{C}$. (It is easy to show that an operator on $\mathscr{A}$ is multiplicative iff it is a composition operator.) In an unpublished set of notes [6] Lindenstrauss, Phelps, and Ryff constructed a nonmultiplicative extreme element of $\mathscr{P}$. Rocherg later put the example in [6] into a much broader setting by considering subsets of $\mathscr{P}$ of the form

$$
K(F, G)=\{T \in \mathscr{P} \mid T F=G\} .
$$

Rochberg proved in [10] that, if $F$ and $G$ are inner functions having $n \geqq 1$ and $m$ zeros respectively (counting multiplicity), then the convex set $K(F, G)$ has real dimension $\leqq(n-1)(m+1)$. In [7] we observed that $[F, G]$ always belongs to $K(F, G)$ and, hence, that $K(F, G) \neq \phi$. It is easy to show that $K(F, G)$ is an extreme subset of $\mathscr{P}$, that is to say, $1 / 2\left(T_{1}+T_{2}\right) \in K(F, G)$ and $T_{1}, T_{2} \in \mathscr{P}$ imply $T_{1}, T_{2} \in K(F, G)$, it follows that every extreme point of $K(F, G)$ is also an extreme point of $\mathscr{P}$. Unless $G$ is of the form $F \circ H$, no element of $K(F, G)$ can be multiplicative. The study of the orbits $\mathscr{P} f$ was begun in [8]. Intuitively, the sets $\mathscr{P} f$ are "cross sections" of the convex set $\mathscr{P}$. We have shown that $\mathscr{P} f=\mathscr{Q}$ iff $f$ is a nonconstant inner function [8]. The main result of this paper can be interpreted as follows: Let $\mathscr{P}_{0}$ denote the weak operator closed convex hull of the operators $[F, G]$ where $F$ and $G$ are inner functions. Then $\mathscr{P}_{0}$ and $\mathscr{P}$ have the same "cross sections". Whether $\mathscr{P}_{0}=\mathscr{P}$ or $\mathscr{P}_{0} \neq \mathscr{P}$ is an open question.

2. The main result. We begin by stating Fisher's theorem from [3].

THEOREM 2.1. Q is the closed convex hull of the inner functions in $\mathscr{A}$.

THEOREM 2.2. For each $f \in \mathscr{U}$, we have

$$
\mathscr{P} f=\overline{\operatorname{cov}}\{[F, G] f \mid F, G \text { inner, } F \text { nonconstant }\} .
$$

Proof. Let $\mathscr{U}_{0}=\{f \in \mathscr{U} \mid$ (1) holds $\}$. It is easy to show that $\mathscr{U}_{0}$ is closed and convex. By Theorem 2.1 it follows that, if $\mathscr{U}_{0}$ contains every inner function, then $\mathscr{U}_{0}=\mathscr{C}$. Clearly $\mathscr{U}_{0}$ contains the constant inner functions. Suppose that $f$ is inner and nonconstant and that $T$ is an operator in $\mathscr{P}$ such that 


\section{$T f \notin \overline{\operatorname{cov}}\{[F, G] f \mid F, G$ inner, $F$ nonconstant $\}$.}

By the Hahn-Banach theorem there exists a bounded linear functional $\ell$ on $\mathscr{A}$ such that

(2) $\sup \{\operatorname{Re} \ell([F, G] f) \mid F, G$ inner, $F$ nonconstant $\}<\operatorname{Re} \ell(T f)$.

Since $T f \in \mathscr{U}$, it follows from, Theorem 2.1 that, given $\varepsilon>0$ there exists an inner function $g$ such that

$$
\operatorname{Re} \iota(T f)-\varepsilon \leqq \operatorname{Re} \iota(g)=\operatorname{Re} \iota([f, g] f) .
$$

But if $\varepsilon$ is sufficiently small then (2) is contradicted.

Of course Theorem 2.1 is crucial in the proof of Theorem 2.2. We will now show that Theorem 2.1 can be derived from Theorem 2.2 .

Proposition 2.3. Let $Z$ denote the identity on $\bar{D}$. Let $F$ and $G$ be inner functions in $\mathscr{A}$ with $F$ nonconstant. Then the functian $[F, G] Z$ is a convex combination of at most four inner functions.

Proof. Let $n$ denote the number of zeros of $F$ (counting multiplicity) and let $\alpha=F(0)$. Consider the linear fractional transformation $h_{\alpha}(w)=(w-\alpha) /(1-\bar{\alpha} w)$. Note that $h_{\alpha} \circ F$ is of the form $Z F_{1}$, where $F_{1} \in \mathscr{A}$. Since $h_{\alpha}$ maps $\partial D$ homeomorphically onto itself, it follows that every continuous function $g$ on $\partial D$ can be uniformly approximated by polynomials in $h_{\alpha}$ and $\bar{h}_{\alpha}$. Thus, if it is known that both $g$ and $h_{\alpha} \bar{g}$ belong to $\mathscr{A} \mid \partial D$, then it follows that there are constants $a$ and $b$ such that $g=a+b h_{\alpha}$. Let $g=[F, Z] Z \mid \partial D$. Then, if $\Gamma$ is an appropriately chosen circle we have,

$$
g(w)=(2 \pi i n)^{-1} \int_{\Gamma} \xi F^{\prime}(\xi)(F(\xi)-w)^{-1} d \xi=n^{-1} \sum_{F(u)=w} u .
$$

Thus,

$$
\begin{aligned}
\left.h_{\alpha}(w) \overline{g(w}\right) & =n^{-1} \sum_{F(u)=w} h_{\alpha}(w) \bar{u} \\
& =n^{-1} \sum_{F(u)=w} u F_{1}(u) \bar{u} \\
& =\left([F, Z] F_{1}\right)(w) .
\end{aligned}
$$

Thus, it follows that $h_{\alpha} \bar{g} \in \mathscr{A} \mid \partial D$. Hence, we may write

$$
\begin{aligned}
{[F, G] Z } & =([F, Z] Z) \circ G \\
& =a+b h_{\alpha} \circ G .
\end{aligned}
$$

Since $\|[F, G] Z\| \leqq 1$, it follows that $|a|+|b| \leqq 1$. Let $e^{2 s}=a /|a|$ 
and $e^{i t}=b /|b|$. Then

$$
\begin{gathered}
{[F, G] Z=e^{i s}|a|+e^{i t}|b| h_{\alpha} \circ G+\frac{1}{2}(1-|a|-|b|) Z} \\
+\frac{1}{2}(1-|a|-|b|)(-Z) .
\end{gathered}
$$

To derive Theorem 2.1 from Theorem 2.2 we argue as follows: for each $h \in \mathscr{Q}$ we have $h=C_{h} Z \in \mathscr{P} Z$. By Theorem 2.2, for each $\varepsilon>0$ there exist inner functions $F_{1}, F_{2}, \cdots, F_{m}, G_{1}, G_{2}, \cdots, G_{m}$ and constants $c_{1}, c_{2}, \cdots, c_{m}$, where the $F_{i}$ 's are nonconstant, where the $c_{i}$ 's are nonnegative, and where $\sum c_{i}=1$, such that

$$
|| h-\sum_{i=1}^{m} c_{i}\left[F_{i}, G_{i}\right] Z \mid<\varepsilon .
$$

It follows from Proposition 2.3 that $\sum c_{i}\left[F_{i}, G_{i}\right] Z$ is a convex combination of inner functions.

3. Possible extension to $H^{\infty}$. It is tempting to try to prove an analogue of Theorem 2.2 for the algebra $H^{\circ}$. Certainly infinite Blaschke products can replace the finite ones and a replacement for Theorem 2.1 exists, namely, the result of Bernard, Garnett, and Marshall [2, Th 2.2] which implies that the unit ball in $H^{\infty}$ is the closed convex hull of the Blaschke products. The difficulty lies in finding appropriate analogues for the operators $[F, G]$. It is not even clear that, given infinite Blaschke products $B_{1}$ and $B_{2}$, there exists a linear operator $T: H^{\infty} \rightarrow H^{\infty}$ such that $\|T\|=T 1=1$ and $T B_{1}=B_{2}$. All that we have been able to accomplish is to find a sufficient condition on $B_{1}$ in order that $T$ exist.

Let

$$
\mathscr{P}\left(H^{\infty}\right)=\left\{T: H^{\infty} \longrightarrow H^{\infty} \mid T \text { linear, }\|T\|=T 1=1\right\} .
$$

$\mathscr{P}\left(H^{\infty}\right)$ carries a locally convex topology $\tau$ which is defined in terms of nets by: $T_{d} \stackrel{\tau}{\rightarrow} T$ if $T_{d} f$ converges uniformly on compact subsets of $D$ to $T f$ for each $f \in H^{\infty}$. By a result due to Kadison [5], it follows that $\mathscr{P}\left(H^{\infty}\right)$ is $\tau$-compact.

Proposition 3.1. Let $g$ be a nonconstant member of $H^{\infty}$ with $\|g\| \leqq 1$. Let

$$
B(z)=\prod_{k=1}^{\infty} \frac{\left|\alpha_{k}\right|}{\alpha_{k}} \cdot \frac{\alpha_{k}-z}{1-\bar{\alpha}_{k} z}
$$

be a convergent Blaschke product. Let 


$$
B_{n}(z)=\prod_{k=1}^{n} \frac{\left|\alpha_{k}\right|}{\alpha_{k}} \cdot \frac{\alpha_{k}-z}{1-\bar{\alpha}_{k} z}
$$

and let $\widetilde{B}_{n}=B / B_{n}$. If the following condition is satisfied then there exists a $T \in \mathscr{P}\left(H^{\infty}\right)$ such that $T B=g$ :

(C) Some subsequence of $\left\{n^{-1} \sum_{k=1}^{n} \widetilde{B}_{n}\left(\alpha_{k}\right)\right\}_{n=1}^{\infty}$ converges to 1 .

Proof. First we show that it is enough to consider the case where $g=Z$. Since $g$ is nonconstant, the composition operator $C_{g}$ is well defined on $H^{\infty}$. If $S$ maps $B$ to $Z$, then $C_{g} \circ S$ will map $B$ to $g$. For each $n$, the operator $T_{n}=\left[B_{n}, Z\right]$ extends to an operator on $H^{\infty}$ via the formula

$$
T_{n} f(z)=(2 \pi i n)^{-1} \int_{\partial D} f(\xi) B_{n}^{\prime}(\xi)\left(z-B_{n}(\xi)\right)^{-1} d \xi,
$$

for $z \in D$. (Here we are using the fact that functions in $H^{\infty}$ have radial limits almost everywhere on $\partial D . \quad f(\xi)$ denotes $\lim _{r \rightarrow 1} f(r \xi)$.) Let $\left\{n_{k}\right\}$ be a sequence of integers such that

$$
\lim _{k} n_{k}^{-1} \sum_{j=1}^{n_{k}} \widetilde{B}_{n_{k}}\left(\alpha_{j}\right)=1 \text {. }
$$

Consider

$$
\begin{aligned}
T_{n_{k}} B(w)-w & =n_{k}^{-1}\left(\sum_{B_{n_{k}}(u)=w} B(u)-w\right. \\
& =n_{k}^{-1}\left(\sum_{B_{n_{k}}(u)=w} w^{\widetilde{B}_{n_{k}}}(u)\right)-w \\
& =w\left(\left(T_{n_{k}} \widetilde{B}_{n_{k}}\right)(w)-1\right) .
\end{aligned}
$$

The function $g_{k}=T_{n_{k}} \widetilde{B}_{n_{k}}-1$ is zero free for each $k$. Let $g_{k_{f}}$ be a subsequence of $g_{k}$ which converges uniformly on compact subsets of $D$ to $g$. By a theorem due to Hurwitz [1, p. 178] $g$ is either identically zero in $D$ or has no zeros in $D$. By (3) it follows that $g(z) \equiv 0$. We have constructed a sequence of integers $m_{j}$ such that $T_{m_{j}} B$ converges uniformly on compact subsets of $D$ to $Z$. It follows easily from the $\tau$-compactness of $\mathscr{P}\left(H^{\infty}\right)$ that there is a $T \in \mathscr{P}\left(H^{\infty}\right)$ with $T B=Z$.

COROLlary 3.2. Let $g$ be an extreme element of the closed unit ball of $H^{\infty}$. Let $B$ be an infinite Blaschke product satisfying (C). Then there exists an extreme element $T_{0}$ of $\mathscr{P}\left(H^{\infty}\right)$ such that $T_{0} B=g$.

Proof. The set $f=\left\{T \in \mathscr{P}\left(H^{\infty}\right) \mid T B=g\right\}$ is convex, $\tau$-closed and nonempty. It follows from the Krein-Milman theorem that $f$ 
has an extreme point $T_{0}$. Since $\mathscr{F}$ is an extreme subset of $\mathscr{P}\left(H^{\infty}\right)$, it follows that $T_{0}$ is also an extreme point of $\mathscr{P}\left(H^{\infty}\right)$.

REMARKS. Note that

$$
1-n^{-1} \sum_{k=1}^{n} \widetilde{B}_{n}\left(\alpha_{k}\right)=n^{-1} \sum_{k=1}^{n} \sum_{\mathfrak{l}=n}^{\infty} \widetilde{B}_{\mathfrak{t}+1}\left(\alpha_{k}\right)-\widetilde{B}_{\mathfrak{t}}\left(\alpha_{k}\right) .
$$

It follows that

$$
\begin{aligned}
& \left|1-n^{-1} \sum_{k=1}^{n} \widetilde{B}_{n}\left(\alpha_{k}\right)\right| \leqq n^{-1} \sum_{k=1}^{n} \sum_{\mathfrak{l}=n}^{\infty}\left|1-\frac{\left|\alpha_{\mathfrak{\imath}}\right|}{\alpha_{\mathfrak{\imath}}} \cdot \frac{\alpha_{\mathfrak{l}}-\alpha_{k}}{1-\bar{\alpha}_{\mathfrak{\imath}} \alpha_{k}}\right| \\
& \leqq 2 n^{-1} \sum_{k=1}^{n} \sum_{\mathfrak{l}=n}^{\infty} \frac{1-\left|\alpha_{\mathfrak{l}}\right|}{\left|1-\bar{\alpha}_{\mathfrak{\imath}} \alpha_{k}\right|} .
\end{aligned}
$$

Hence, condition (C) is implied by the following:

$$
\lim \inf n^{-1} \sum_{k=1}^{n} \sum_{\mathfrak{l}=n}^{\infty} \frac{1-\left|\alpha_{\mathfrak{r}}\right|}{\left|1-\bar{\alpha}_{\mathfrak{r}} \alpha_{k}\right|}=0 .
$$

Now suppose that the zeros of $B$ approach $\partial D$ exponentially fast. To be precise suppose that there is a $b \in(0,1)$ such that $\left(1-\left|\alpha_{n}\right|\right) \mid$ $\left(1-\left|\alpha_{n-1}\right|\right) \leqq b$ for $n=2,3, \cdots$. Then for $\mathfrak{l}>k$ we have

$$
\frac{1-\left|\alpha_{\mathfrak{\imath}}\right|}{\left|1-\bar{\alpha}_{\mathfrak{r}} \alpha_{k}\right|} \leqq \frac{1-\left|\alpha_{\mathfrak{r}}\right|}{1-\left|\alpha_{k}\right|} \leqq b^{\mathrm{r}-k}
$$

Condition (C) follows immediately. On the other hand, if the zeros of $B$ approach $\partial D$ too slowly, condition (C) may fail to hold. Consider the case where $\alpha_{k}=1-k^{-p}$, where $p>1$. For $k \leqq n$ we have

$$
1-\frac{k^{-p}-(n+1)^{-p}}{k^{-p}+(n+1)^{-p}-k^{-p}(n+1)^{-p}} \leqq 1-\widetilde{B}_{n}\left(\alpha_{k}\right) .
$$

It follows that

$$
1-\sum_{k=1}^{n} \frac{1}{n} \frac{1-(k /(n+1))^{p}}{1+(k /(n+1))^{p}-(n+1)^{-p}} \leqq 1-n^{-1} \sum_{k=1}^{n} \widetilde{B}_{n}\left(\alpha_{k}\right) .
$$

Thus,

$$
0<1-\int_{0}^{1} \frac{1-x^{p}}{1+x^{p}} d x \leqq \lim \inf \left(1-n^{-1} \sum_{k=1}^{n} \widetilde{B}_{n}\left(\alpha_{k}\right)\right) .
$$

We note that in this example $\left(\alpha_{k}\right)$ is not an interpolating sequence. See [4]. We have been unable to determine whether or not condition (C) holds whenever $\left(\alpha_{k}\right)$ is an interpolating sequence. 


\section{REFERENCES}

1. L. Ahlfors, Complex Analysis 3rd ed., McGraw-Hill, 1979.

2. A. Bernard, J. B. Garnett, D. E. Marshall, Algebras generated by inner functions, J. of Functional Analysis, 25 (1977), 275-285.

3. S. Fisher, The convex hull of the finite Blaschke products, Bull. A.M.S., 74 No. 6, Nov. 1966.

4. K. Hoffmann, Banach Spaces of Analytic Functions, Prentice-Hall, 1962.

5. R. V. Kadison, The trace in finite operator algebras, Proc. A.M.S., 12 (1961).

6. J. Lindenstrauss, R. Phelps J. V. Ryff, Extreme nonmultiplicative operators, lecture notes, University of Washington, Seattle, Washington.

7. J. N. McDonald, Convex sets of operators on the disk algebra, Duke Math. J., 42 No. 4, (Dec. 1975), 787-796.

8. - Positive operators on the disk algebra, Indiana U. Math. J., 26 No. 6 (1977), 1057-1065.

9. R. B. Phelps, Extreme positive operators and homomorphisms, Trans. A.M.S., 108 (1963), 265-274.

10. R. Rochberg, Linear maps of the disk algebra, Pacific J. Math., 44 No. 1 (1973).

Received March 4, 1980 and in revised form September 22, 1980.

Arizona State University

TEMPE, AZ 85281 



\section{PACIFIC JOURNAL OF MATHEMATICS}

\section{EDITORS}

DONALD BABBITT (Managing Editor)

University of California

Los Angeles, California 90024

Hugo RossI

University of Utah

Salt Lake City, UT 84112

C. C. MOORE and ANDREW OGG

University of California

Berkeley, CA 94720

\section{J. DugundJI}

Department of Mathematics University of Southern California Los Angeles, California 90007

R. Finn and J. Milgram Stanford University

Stanford, California 94305

\section{ASSOCIATE EDITORS}
R. ARENS
E. F. BeCKENBACH
B. H. Neumann
F. WoLF
K. YoSHIDA

\section{SUPPORTING INSTITUTIONS}

UNIVERSITY OF ARIZONA

UNIVERSITY OF BRITISH COLUMBIA

CALIFORNIA INSTITUTE OF TECHNOLOGY

UNIVERSITY OF CALIFORNIA

MONTANA STATE UNIVERSITY

UNIVERSITY OF NEVADA, RENO

NEW MEXICO STATE UNIVERSITY

OREGON STATE UNIVERSITY
UNIVERSITY OF OREGON

UNIVERSITY OF SOUTHERN CALIFORNIA

STANFORD UNIVERSITY

UNIVERSITY OF HAWAII

UNIVERSITY OF TOKYO

UNIVERSITY OF UTAH

WASHINGTON STATE UNIVERSITY

UNIVERSITY OF WASHINGTON 


\section{Pacific Journal of Mathematics}

\section{Vol. 97, No. $1 \quad$ January, 1981}

Charles A. Asmuth and Joe Repka, Tensor products for $S L_{2}(\mathfrak{k})$. II.

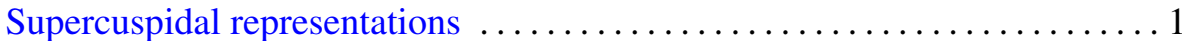

Joseph Barback, On finite sums of regressive isols . ................. 19

Matthew G. Brin and Daniel Russell McMillan, Jr., Generalized

three-manifolds with zero-dimensional nonmanifold set ............29

Kun Soo Chang, Converse measurability theorems for Yeh-Wiener space . . . 59

Christopher Brian Croke, A "maximal torus" type theorem for complete

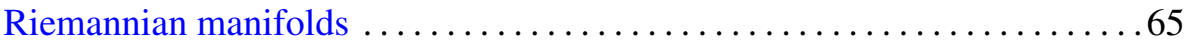

Gustave Adam Efroymson, Sums of squares in planar Nash rings . . . . . . 75

John Robert Fisher, Axiomatic radical and semisimple classes of rings . . . .81

Betty Kvarda, Consecutive integers for which $n^{2}+1$ is composite .......93

Roosevelt Gentry, New diagram proofs of the Hausdorff-Young theorem

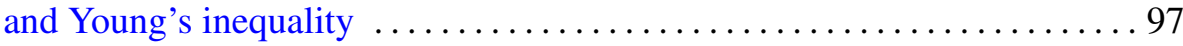

Patrick M. Gilmer, Topological proof of the $G$-signature theorem for $G$

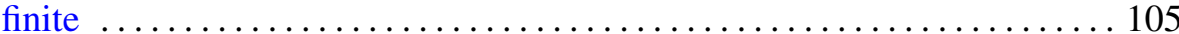

Chung Wei Ha, A noncompact minimax theorem .................. 115

James J. Hebda, Manifolds admitting taut hyperspheres ................ 119

Takayuki Kawada, Sample functions of Pólya processes ............. 125

Peter K. F. Kuhfittig, Common fixed points of nonexpansive mappings by

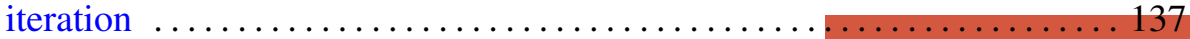

James Thomas Loats and Judith Roitman, Almost rigid Hopfian and dual

Hopfian atomic Boolean algebras .......................... 141

Roger McCann, On embedding semiflows into a radial flow on $l_{2} \ldots \ldots \ldots 151$

John McDonald, Closed orbits of convex sets of operators on the disk

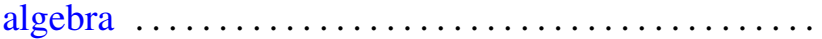

Mark D. Meyerson, Convexity and the table theorem .............. 167

Arnold William Miller, Generic Souslin sets . . . . . . . . . . . . . . . . 171

Takemi Mizokami, On the closed images of paracomplexes $\ldots \ldots \ldots \ldots \ldots 3$

Jagannadham Venkata Pakala and Thomas Stephen Shores, On

compactly packed rings $\ldots \ldots \ldots \ldots \ldots \ldots \ldots \ldots \ldots$

Andrew Pletch, Strong completeness in profinite groups

Wilbur Carrington Whitten, Inverting double knots

James Juei-Chin Yeh, Existence of strong solutions for stochastic

differential equations in the plane 